\title{
Hippocampal Damage Equally Impairs Memory for Single Items and Memory for Conjunctions
}

\author{
Craig E.L. Stark ${ }^{1}$ and Larry R. Squire ${ }^{2 *}$ \\ ${ }^{1}$ Departments of Psychological and Brain Sciences and \\ Neuroscience, Johns Hopkins University, \\ Baltimore, Maryland \\ ${ }^{2}$ Veterans Affairs Medical Center and Departments of \\ Psychiatry, Neurosciences and Psychology, University of \\ California, San Diego, California
}

\begin{abstract}
In a prior study of continuous recognition performance, data were reported in support of the hypothesis that the hippocampus is not needed to remember the individual components of a stimulus but is important for remembering associations between its components (Kroll et al. 1996. J Mem Lang 35:176-196). Patients with left hippocampal damage were able to endorse recently encountered words and to reject novel words, as well as disyllabic words in which one of the syllables had been previously encountered. However, they failed to reject words in which both syllables had been encountered independently in different words. We present data from five experiments designed to examine this finding in more detail. In each experiment, five patients with bilateral hippocampal damage and eight controls were tested using the same protocol as Kroll et al. (1996). On each trial, a two-component stimulus was presented. Stimuli could be entirely novel, novel with one previously encountered (repeated) component, novel but with both components repeated, or a true repetition. The first experiment was a direct replication using the same disyllabic words as Kroll et al. (1996). The second experiment used pseudo-words, constructed of two monosyllabic words (e.g., jambark). The third experiment used the same pairs of monosyllabic words, but presented separately on the screen to encourage participants to treat each component independently. The fourth experiment used pairs of objects, and the fifth experiment used face-house pairs. In all five experiments, patients with hippocampal damage exhibited impaired recognition memory. The impairment extended across all trial types with no evidence that hippocampal damage selectively (or disproportionately) impaired the associative or conjunctive component of memory. We discuss our findings in the light of the work by Kroll et al. (1996) and other recent neuropsychological, electrophysiological, and neuroimaging studies of hippocampal function and single-item and associative memory. Hippocampus 2003; 13:281-292. ๑ 2003 Wiley-Liss, Inc.
\end{abstract}

KEY WORDS: hippocampus; association; amnesia; configuration; medial temporal lobe

Grant sponsor: Medical Research Service of the Department of Veterans Affairs; Grant sponsor: National Institute of Mental Health; Grant number: MH24600; Grant number: MH12278; Grant sponsor: National Alliance for Research on Schizophrenia and Depression (NARSAD); Grant sponsor: Metropolitan Life Foundation.

*Correspondence to: Larry R. Squire, Veterans Affairs Medical Center, 116-A, 3350 La Jolla Village Drive, San Diego, CA 92161.

E-mail: Isquire@ucsd.edu

Accepted for publication 3 June 2002

DOI 10.1002/hipo.10085

\section{INTRODUCTION}

Although it is established that structures in the medial temporal lobes are essential for the formation of declarative memory (memory for facts and events), the contribution of the separate components of the medial temporal lobe (the hippocampus proper, the dentate gyrus, the subiculum, and the entorhinal, perirhinal, and parahippocampal cortices) remains unclear. Particular attention has been focused on the role of the hippocampus in declarative memory. One hypothesis is that, relative to the cortical structures in the medial temporal lobe, the hippocampus plays an especially significant role in "associative" or "conjunctive" memory (Sutherland and Rudy, 1989; Eichenbaum et al., 1994; Vargha-Khadem et al., 1997; Henke et al., 1997, Henke et al., 1999; Aggleton and Brown, 1999; Eldridge et al., 2000; Brown and Aggleton, 2001). In the strongest version of this hypothesis, the hippocampus does not contribute to performance on traditional recognition memory tasks unless the tasks include an explicitly recollective or associative component. Another view is that the hippocampus and the adjacent structures of the medial temporal lobe (the parahippocampal gyrus) are broadly important for declarative memory (Reed and Squire, 1999; Stark and Squire, 2001a; Broadbent et al., 2002). According to this view, all tasks of declarative memory, including familiarity-based recognition, depend on forming associations, and the hippocampus as well as the parahippocampal gyrus play a role in associative memory. By this view, a simple distinction between associative and nonassociative memory does not capture the division of labor between the hippocampus and adjacent structures, and associative memory or memory for conjunctions has no special status with respect to the hippocampus.

In a study of association learning, Kroll et al. (1996, Experiment 1) reported data that were consistent with a strong view of the first hypothesis - that the hippocam- 


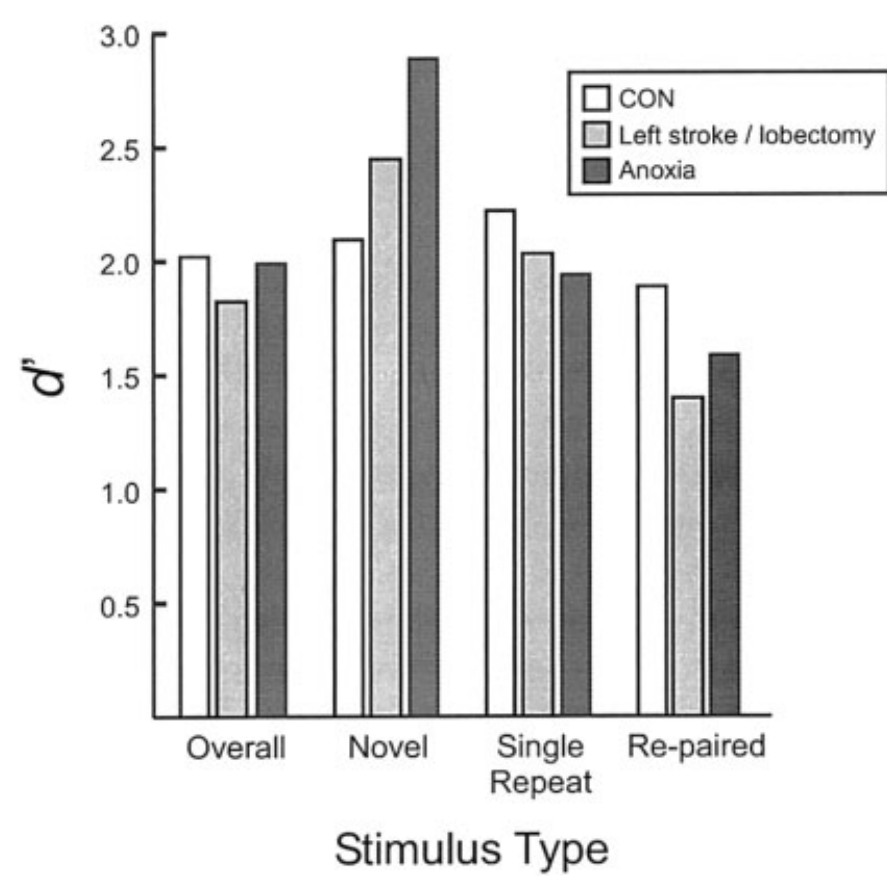

FIGURE 1. Data from Exp.1 of Kroll et al. (1996) showing performance on a continuous recognition memory task designed to assess associative and nonassociative components of memory. Overall discriminability $\left(d^{\prime}\right)$ scores (Overall) were calculated from the hit rate (probability of responding "yes" to true repetitions) and the overall false alarm rate (probability of responding "yes" to all other items) for age-matched controls ( $\mathrm{CON} ; \mathrm{n}=18)$, patients with damage to the left medial temporal lobe (Left stroke/lobectomy; $\mathbf{n}=7$ ), and a patient with bilateral medial temporal lobe damage (Anoxia; $n=1$ ). In addition, $d^{\prime}$ scores were calculated for each of the three possible false alarm conditions: entirely novel words (Novel), novel words in which one syllable had been presented previously (Single Repeat), and novel words in which both syllables had been presented previously (Repaired). These three conditions correspond to the "First word," "Syllable," and "Conjunction" conditions in Kroll et al. (1996). The critical finding in the study by Kroll et al. (1996) was a selective impairment for Re-paired trials in the patients.

pus is especially important for forming associations within declarative memory. In the study, both controls and patients with damage to the hippocampus were given a continuous recognition memory task and were asked to respond "yes" when a previously encountered word appeared. The stimuli used in the task were disyllabic concrete nouns that could appear in one of four conditions: novel words that had not appeared earlier in the list, novel words that shared a single syllable with a previously encountered word, novel words in which both syllables had appeared in different previously encountered words, or words that had been previously encountered.

The results from the experiment, converted into discriminability $\left(d^{\prime}\right)$ scores, are shown in Figure 1. The finding of interest is that patients with damage to either the left hippocampus (Left stroke/ lobectomy) or to both the left and right hippocampus (Anoxia) were impaired only on trials in which the word was a re-pairing of two previously presented syllables (Re-paired). Both syllables in the Re-paired words were familiar, and patients with hippocampal damage tended to respond "yes" to the recognition probe, as if they were unable to identify these words as novel combinations of familiar components. These findings are supportive of the hypothesis that damage to the hippocampal region does not impair memory for the individual components of a stimulus (e.g., the syllables) but does impair memory for associations between the components. As a result, patients could not remember whether the two syllables of a word had appeared together earlier in the same word or whether the syllables had appeared as parts of two different words.

One notable aspect of the data in this earlier study is that the patients performed numerically better than their age-matched controls when they encountered stimuli that would constitute a traditional recognition memory test. Thus, Kroll et al. (1996) reported that age-matched controls scored $69.4 \%$ correct on standard recognition (hit rate for repeated words minus the false alarm rate for entirely novel words), whereas the seven patients with unilateral left temporal lobe damage scored $77.2 \%$ correct and the one patient with bilateral damage scored $84.0 \%$ correct. Discriminability $\left(d^{\prime}\right)$ scores for these three groups are shown in Figure 1 and illustrate that simple recognition accuracy (novel bars) was numerically higher in the patient groups than in the control group. Indeed, Kroll et al. (1996) noted that, under the testing conditions they used, the "ability [of the patients] to recognize the true repetitions and to reject completely new words is very similar to that of the subjects without hippocampal damage" (Kroll et al., 1996, p 184).

Yet, amnesic patients with bilateral damage restricted to the hippocampal region are typically impaired in traditional recognition memory tests (Rempel-Clower et al., 1996; Reed and Squire, 1997; Manns and Squire, 1999; Stark and Squire, 2000a). In one case known to us of spared recognition memory in an adult amnesic patient with bilateral hippocampal damage (patient Y.R., Holdstock et al., 2002), recognition performance was spared only in some conditions (e.g., some forced-choice tasks), but was impaired in yes/no tasks in which the foil distracters were similar to targets (but see Reed and Squire, 1997; Stark and Squire, 2000a, for evidence of impaired performance in forced-choice tasks in patients with restricted hippocampal damage). The paradigm employed by Kroll et al. (1996) would therefore seem to be the kind of task that even patient Y.R. would find difficult, as the paradigm involves endorsing repeated items with a "yes" response in the face of similar distracters. One would therefore expect that bilateral hippocampal damage, at least in the case of adult-onset amnesia, should result in impaired (and certainly not enhanced) recognition memory performance (For reports of good recognition performance in cases of developmental early-onset amnesia, see VarghaKhadem et al., 1997; Baddeley et al., 2001).

Another possible concern is that the controls tested by Kroll et al. (1996) may have performed unusually poorly (potentially resulting from an inadvertent mismatch in factors such as the amount of education). For example, note in Figure 1 that, if the scores of the controls were elevated across all conditions, evidence for a selective impairment in the Re-paired condition would be weakened, and evidence would grow for an impairment in the Single Repeat condition as well. It is also noteworthy that the patients in the "left stroke lobectomy" group all had damage outside the hippocampus, including damage to the parahippocampal gyrus (stroke patients) or damage to the parahippocampal gyrus 

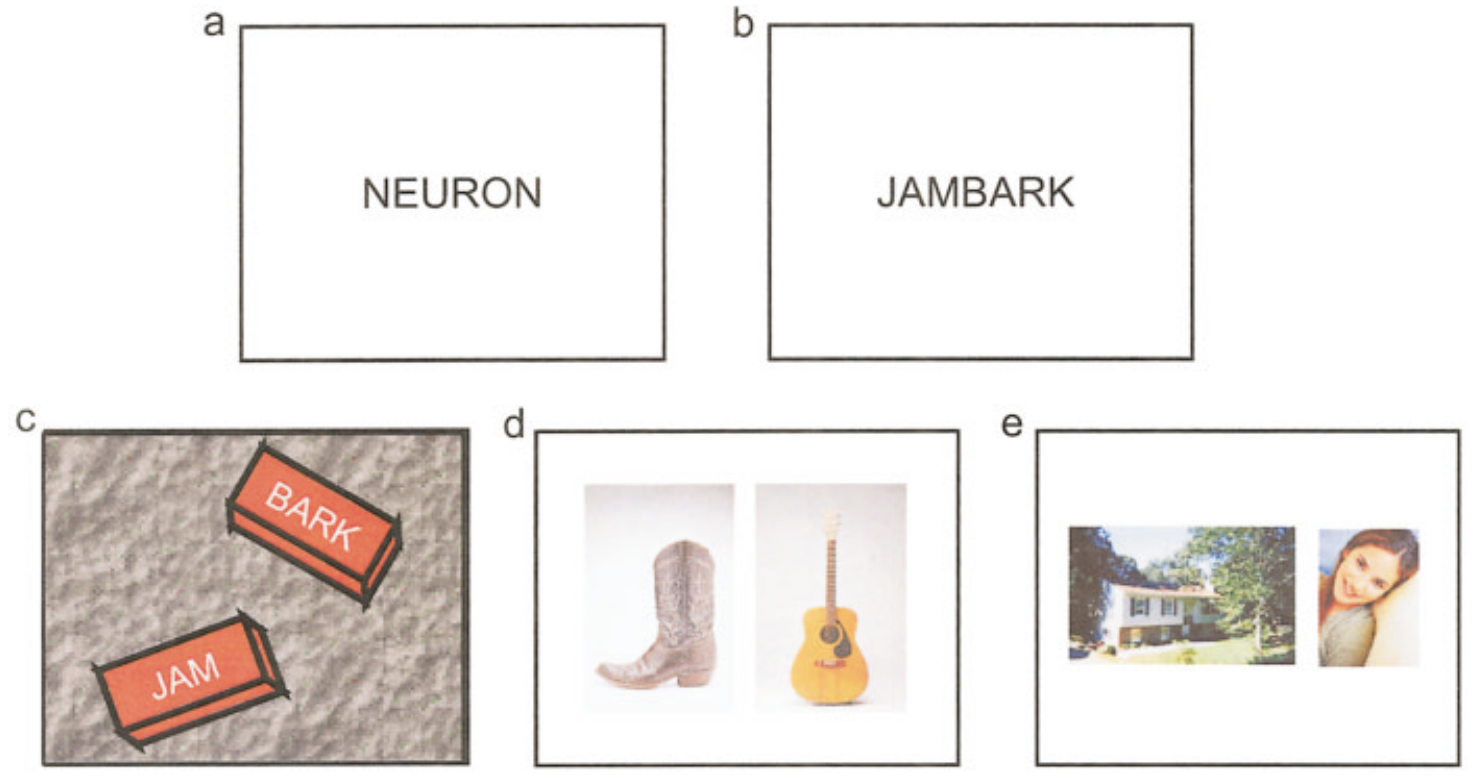

FIGURE 2. Sample stimuli from Experiments 1-5. In all five experiments, a two-component stimulus was presented on each trial as part of a continuous recognition task. Stimuli could be entirely novel (neither component had been encountered previously); one component could have been encountered previously; both components could have been encountered previously as part of different stimuli; or the stimulus could be a true repetition of a previously encountered item. A selective impairment for stimuli that are novel but composed

and temporal lobe neocortex (lobectomy patients). The fact that these lesions extended beyond the hippocampus makes it difficult to attribute poor performance (e.g., in a Re-paired condition) to the hippocampus itself. In addition, it is not straightforward to predict the nature of memory impairment in the case of unilateral damage, because patients approach the task with both intact and impaired memory systems. Finally, it is also worth noting that the patient with bilateral damage may have had damage beyond the hippocampus (a possibility consistent with the report that this patient exhibited the most severe anterograde amnesia of any of the patients tested). Thus, although the results obtained by Kroll et al. (1996) are intriguing, the possibility that the controls performed poorly, and the fact that the lesions were mainly unilateral and extended beyond the hippocampus, make it difficult to draw firm conclusions from this study about the role of the hippocampus itself in associative memory.

In the present study, we report the results from five experiments designed to explore the effect of hippocampal damage on recognition memory. All five experiments used the same continuous recognition paradigm employed by Kroll et al. (1996) and evaluated the performance of five patients with bilateral damage to the hippocampal region. The first experiment attempted to repeat the procedure from Experiment 1 of Kroll et al. (1996). Experiments 2-5 attempted to increase progressively the degree to which associative memory would be used to perform the task (Fig. 2). In all five experiments, we found that the impairment in patients with hippocampal damage was not limited to the Re-paired trials but extended across all trial types. of previously encountered components would indicate a specific impairment in forming associations. a: Stimuli in Experiment 1, which repeated the procedure of Kroll et al. (1996) using disyllabic nouns. b: Stimuli in Experiment 2, which used pseudo-words comprised of monosyllabic nouns. c: Stimuli in Experiment 3, which used the same monosyllabic nouns but presented in isolation. d: Stimuli in Experiment 4, which used two color pictures of common objects. e: Stimuli in Experiment 5, which used pictures of houses and people.

Thus, we found no evidence that memory impairment was disproportionately weighted toward an associative (or conjunctive) component of declarative memory.

\section{EXPERIMENT 1}

Experiment 1 repeated the first experiment conducted by Kroll et al. (1996). Amnesic patients with damage to the hippocampal region (CA fields, dentate gyrus, and subiculum) and healthy control volunteers were given a continuous recognition memory task involving 219 disyllabic words. Each word belonged to one of four conditions: the word could be novel in that it had not been presented yet in the list (Novel), the word could be novel but have one syllable in common with a previously encountered word (Single Repeat), the word could be novel but have both syllables in common with two different previously encountered words (Re-paired), or the word could be a repeated item (True Repetition). If patients with damage to the hippocampus have a specific impairment in their ability to form explicit associations within declarative memory, accuracy for the Re-paired items should be differentially impaired. Specifically, as reported in Kroll et al. (1996), the patients with damage to the hippocampus should exhibit a greater reduction in accuracy for the Re-paired trials than for trials that do not involve an associative component (e.g., the Single Repeat trials). 
TABLE 1.

Characteristics of Amnesic Patients*

\begin{tabular}{lcccccccr}
\hline & & & & \multicolumn{5}{c}{ WMS-R } \\
\cline { 5 - 9 } Patient & Age & Education & WAIS-III IQ & Attention & Verbal & Visual & General & Delay \\
\hline A.B. & 62 & 20 & 107 & 87 & 62 & 72 & 54 & $<50$ \\
H.C. & 38 & 20 & 98 & 96 & 83 & 53 & 68 & 51 \\
L.J. & 62 & 12 & 101 & 105 & 83 & 60 & 69 & $<50$ \\
M.J. & 61 & 16 & 139 & 125 & 62 & 93 & 62 & $<50$ \\
P.H. & 77 & 19 & 115 & 117 & 67 & 83 & 70 & 57
\end{tabular}

*The Wechsler Adult Intelligence Scale-Revised (WAIS-R) and the indices of the Wechsler Memory Scale-Revised (WMS-R) yield a mean score of 100 in the normal population with a standard deviation of 15 . Four of the patients are male; L.J. is female. For additional neuropsychological data, see Manns and Squire (2001).

\section{Method}

\section{Participants}

Five patients with damage to the hippocampal region (CA fields, dentate gyrus, and subiculum) participated in all five experiments: A.B., H.C., L.J., M.J., and P.H (Table 1). For four of the patients (H.C., L.J., M.J., and P.H), magnetic resonance imaging (MRI) quantified the damage to the medial temporal lobe (for details, see Manns and Squire, 2001). All four have reduced hippocampal area bilaterally (reductions of $27 \%, 46 \%, 22 \%$, and $30 \%$, respectively). In addition, the area of the parahippocampal gyrus for H.C. and P.H. is reduced bilaterally by $25 \%$ and $30 \%$, respectively (L.J., $6 \%$; M.J., $-5 \%)$. Patient H.C. underwent a right parietal craniotomy to evacuate a right occipital and parietal hematoma after a ruptured arteriovenous malformation. His memory impairment is thought to have resulted from the ischemia associated with this rupture. L.J. became amnesic during a 6-month period that began in 1988 with no known precipitating event. Her memory impairment has remained stable since that time. Patient M.J. had a 10year history of cardiovascular disease. On June 6, 1996, he awoke from a night's sleep complaining of memory difficulties. His impairment has remained stable since that time. P.H. had a 6-year history of 1-2-min "attacks" (with a possible epileptic basis) that were associated with gastric symptoms and transient memory impairment. In July 1989, he suffered from a series of brief episodes that resulted in marked and persistent memory loss.

The fifth patient (A.B.) suffered an anoxic episode in 1976 following a cardiac arrest. A.B. wears a pacemaker and is ineligible to participate in MRI studies. In computed tomography (CT) scans obtained in 2001, temporal lobe volume appeared normal, and the temporal horns were symmetric and normal in size. The basal ganglia and thalamus also appeared normal. The only focal lesions detected were small bilateral foci in the white matter lateral to the head of the caudate nucleus, which appeared to be old lacunar infarctions. Neurological exam indicated well-circumscribed amnesia. While not conclusive, these findings, together with reports that histologically confirmed damage limited to the hippocampal formation can occur after anoxia (Rempel-Clower et al., 1996), suggest that A.B.'s memory impairment is due to damage within the hippocampal region.

Eight healthy volunteer controls (four men and four women) were also tested. The controls were matched to the patients with respect to age $($ mean $=67.4$ years) and education $($ mean $=15.5$ years).

\section{Materials}

The stimuli were graciously provided by N. Kroll and consisted of 219 disyllabic nouns divided into three lists of 73 words (Fig. 2a). The words in each list were presented in a fixed, pseudorandom order. These were the same words used in Experiment 1 of Kroll et al. (1996). Each word was linked to one of four conditions. One condition (Novel) involved 123 novel words (including 15 filler words). Neither syllable of any Novel word had appeared previously in the list as part of another word. The second condition (Single Repeat) involved 36 words in which one of the two syllables had appeared previously in another word (e.g., fickle ... fiction). The third condition (Re-paired) involved 36 words in which both syllables had appeared previously in two different words (e.g., central ... neuron ... neutral). The final condition (True Repetition) involved 18 words that had appeared in the list previously (e.g. kitchen ... kitchen). In addition, two words were placed at the beginning of each list to reduce primacy effects.

To generate the Single Repeat and Re-paired stimuli, word triplets with overlapping syllables were constructed (e.g., fiction, buckle, and fickle). These words were then placed appropriately in the list so that the words could serve in the four conditions of interest. For example, in one order of appearance (fiction ... buckle ... fickle) these words resulted in Novel, Novel, and Re-paired trials. In an alternate order (fickle ... buckle ... fiction), the same words resulted in Novel, Single Repeat, and Single-Repeat trials. As in Kroll et al. (1996), the retention interval (number of items between the cue word and the test word) could be 10, 20, or 40 items, and the lag (number of items between the two cues for Re-paired trials) could be 1 or 5 . In the case of Re-paired trials, the retention interval was the number of trials between the most recent cue word and the test word. Finally, as in Kroll et al. (1996), we 
TABLE 2.

False Alarm Rates*

\begin{tabular}{|c|c|c|c|c|c|}
\hline \multirow[b]{2}{*}{ Exp. } & \multirow[b]{2}{*}{ Group } & \multicolumn{3}{|c|}{ False alarm condition } & \multirow[b]{2}{*}{ Difference scor } \\
\hline & & Novel & $\begin{array}{l}\text { Single } \\
\text { repeat }\end{array}$ & Re-paired & \\
\hline \multirow[t]{2}{*}{1} & $\mathrm{CON}$ & $3.1 \%(0.9)$ & $4.0 \%(1.8)$ & $15.4 \%(4.6)$ & $11.4 \%(3.8)$ \\
\hline & $\mathrm{H}$ & $11.8 \%(7.6)$ & $15.2 \%(10.2)$ & $22.4 \%(10.2)$ & $7.2 \%(2.3)$ \\
\hline \multirow[t]{2}{*}{2} & $\mathrm{CON}$ & $1.8 \%(0.6)$ & $7.1 \%(2.5)$ & $35.2 \%(8.1)$ & $30.6 \%(6.2)$ \\
\hline & $\mathrm{H}$ & $6.8 \%(4.4)$ & $13.0 \%(5.8)$ & $29.6 \%(6.7)$ & $16.6 \%(3.7)$ \\
\hline \multirow[t]{3}{*}{3} & $\mathrm{CON}$ & $3.8 \%(1.5)$ & $11.4 \%$ & $25.6 \%(9.0)$ & $28.2 \%(5.8)$ \\
\hline & Fast CON & $2.9 \%(1.8)$ & $11.8 \%(5.3)$ & $35.1 \%(8.1)$ & $23.4 \%(3.8)$ \\
\hline & $\mathrm{H}$ & $3.2 \%(1.2)$ & $8.8 \%(3.0)$ & $16.0 \%(5.4)$ & $7.2 \%(2.5)$ \\
\hline \multirow[t]{3}{*}{4} & $\mathrm{CON}$ & $0.4 \%(0.2)$ & $9.9 \%(3.2)$ & $37.9 \%(11.0)$ & $28.0 \%(8.2)$ \\
\hline & Fast CON & $1.0 \%(0.8)$ & $21.1 \%(6.3)$ & $41.4 \%(9.5)$ & $20.2 \%(4.7)$ \\
\hline & $\mathrm{H}$ & $2.2 \%(1.0)$ & $10.8 \%(6.2)$ & $30.4 \%(10.7)$ & $19.6 \%(5.4)$ \\
\hline \multirow[t]{3}{*}{5} & $\mathrm{CON}$ & $4.2 \%(1.8)$ & $18.6 \%(6.7)$ & $41.9 \%(8.9)$ & $23.2 \%(3.7)$ \\
\hline & Fast CON & $4.9 \%(1.6)$ & $22.1 \%(6.9)$ & $44.0 \%(10.3)$ & $21.9 \%(4.9)$ \\
\hline & $\mathrm{H}$ & $6.2 \%(3.7)$ & $16.2 \%(9.7)$ & $25.6 \%(12.0)$ & $9.4 \%(3.1)$ \\
\hline
\end{tabular}

CON, healthy volunteers $(n=8)$ who took the same self-paced test given to the patients; Fast CON, Control volunteers $(n=8)$ whose response times were limited to $2 \mathrm{~s} ; \mathrm{H}$, patients with hippocampal damage $(\mathrm{n}=5)$.

*False alarm rates and the difference score (Re-paired false alarm rate minus Single Repeat false alarm rate) used by Kroll et al. (1996) to assess associative memory for Experiments 1-5. Numbers in parentheses indicate the standard error of the mean. In all five experiments, the difference score was smaller for the patients with hippocampal damage $(\mathrm{H})$ than for the control volunteers $(\mathrm{CON})$.

collapsed across retention interval and lag in order to analyze the data.

\section{Procedure}

Words were presented in capital letters in the middle of a computer screen. For each word, participants were asked to decide whether they had seen the word previously on the list. Participants pressed one of two buttons (labeled "yes" and "no") to indicate their responses. The test was self-paced, and participants were given an opportunity to rest at the end of each 73 -item list. Participants were informed that each 73-item test was an isolated test and that items would not repeat across tests.

\section{Results}

Table 2 shows the false alarm rates for the three potential sources of false alarms (Novel words, Single Repeat words, and Re-paired words) together with the difference score used by Kroll et al. (1996) (Re-paired false alarm rate minus Single Repeat false alarm rate). The Re-paired, false alarm rate was always higher than the Single Repeat false alarm rate, reflecting the greater difficulty of distinguishing repaired words (as opposed to Single Repeat words) from words that were previously presented. In contrast to the findings of Kroll et al. (1966) that patients had higher difference scores than controls, in Experiment 1 this difference score was numeri- cally (but not significantly, $P=0.44$ ) less in the patients with hippocampal damage than in the controls.

Participants varied considerably in their overall endorsement rates, making the difference score difficult to interpret unambiguously. Accordingly, we calculated d' scores both for the entire test (Overall) and separately for each of the three potential sources of false alarms: Novel words, Single Repeat words, and Re-paired words (Fig. 3a). It should be noted that the Overall $\mathrm{d}^{\prime}$ score does not provide a score that can be compared to the score on a traditional continuous recognition test. The Overall $\mathrm{d}^{\prime}$ score is based not only on responses to items that would constitute a traditional recognition memory test (True Repetition words and Novel words) but is also based on responses to foil items that were designed to be similar to target items (Single Repeat words and Repaired words). A score comparable to what would be obtained on a traditional continuous recognition memory test can be found in the $\mathrm{d}^{\prime}$ score for Novel words, which is based on responses to true repetitions and novel words.

Figure 3a shows that the hippocampal patients were impaired overall relative to the controls $(t(11)=3.0, P<0.05)$. Further, this impairment was not limited to the Re-paired trials, because the hippocampal patients were impaired on all three trial types (all $P$-values $<$ $0.05)$. In a repeated measures analysis of variance (ANOVA) no interaction was observed between subject group and trial type $(\mathrm{F}<1)$, indicating that the impairment was similar across trial type. 


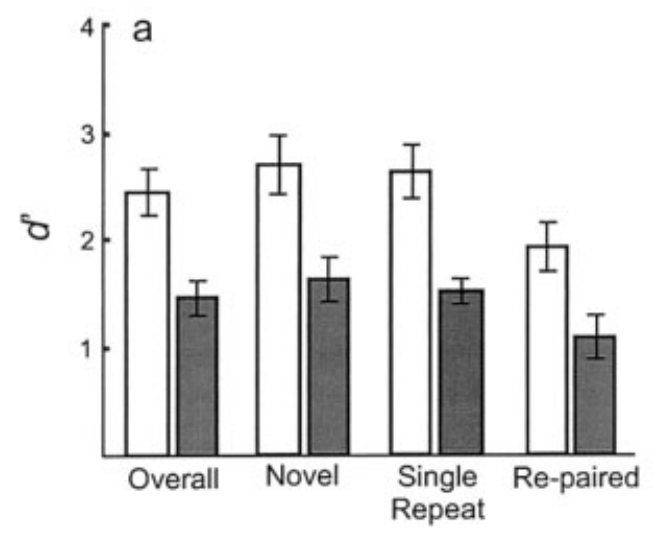

FIGURE 3. Data from Experiments 1 (a) and 2 (b). Overall discriminability $\left(d^{\prime}\right)$ scores (Overall) were calculated from the hit rate (probability of responding "yes" to true repetitions) and the overall false alarm rate (probability of responding "yes" to all other items) for age-matched controls $(\mathrm{CON} ; \mathrm{n}=8)$ and patients with damage to the hippocampal region $(H ; n=5)$. In addition, $d^{\prime}$ scores were calculated using the hit rate and each of the three possible false alarm conditions:

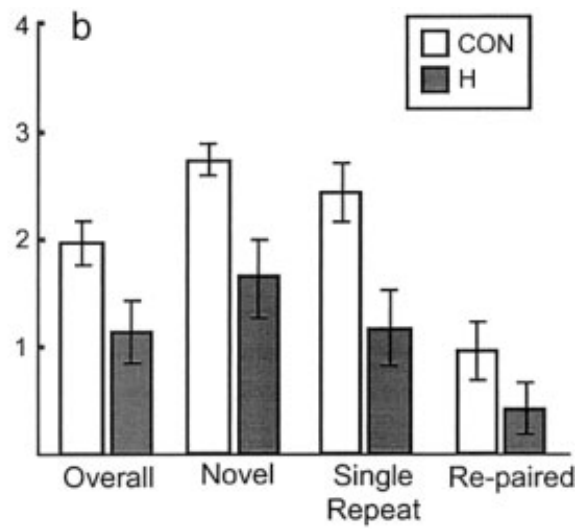

entirely novel stimuli (Novel), stimuli in which one component had been previously presented (Single Repeat), and novel stimuli in which both components had been previously presented but not together (Re-paired). In both experiments, an impairment was observed across all trial types, not only on the Re-paired trials that assess associative memory.

\section{EXPERIMENT 2}

Experiment 1 repeated the procedure of Experiment 1 in Kroll et al. (1996) and asked whether patients with hippocampal damage would be selectively impaired in their memory for associative information. Although patients were impaired overall, the impairment was not more severe on Re-paired trials than on the other trial types. Yet, it seems possible that participants did not treat the disyllabic words as consisting of an association between two different syllables. Participants were very familiar with all the words and therefore may have treated all the words as single entities that had no intrinsic associative component. If so, disyllabic words would not provide an optimal test of whether memory for associations is particularly sensitive to hippocampal damage. In Experiment 2 (and in the experiments that follow), we attempted to make the tasks more explicitly associative. In Experiment 2, disyllabic pseudo-words (e.g., jambark) were used in place of the disyllabic words of Experiment 1 (Fig. 2b).

\section{Method}

Experiment 2 was identical in structure to Experiment 1. The important change was that, instead of disyllabic words, the stimuli consisted of disyllabic pseudo-words (Fig. 2b). The pseudo-words were each comprised of two monosyllabic nouns (e.g., jambark). A new group of eight controls (four men and four women) participated, again matched to the patients with respect to age (mean $=$ 68 years) and education (mean $=16.1$ years).

\section{Results}

Figure $3 \mathrm{~b}$ shows the $\mathrm{d}^{\prime}$ scores for the entire test (Overall) and $\mathrm{d}^{\prime}$ scores based on each of the three potential sources of false alarms (false alarm rates for each condition appear in Table 2).
Overall, the patients with hippocampal lesions were impaired relative to the controls $(\mathrm{t}(11)=2.4, P<0.05)$. A repeated measures ANOVA revealed an interaction between subject group and trial type $(\mathrm{F}(2,22)=4.3, P<0.05)$. However, this interaction resulted from a relative sparing of performance on the Re-paired trials. That is, unlike performance on the Novel trials $(\mathrm{t}(11)=3.2, P<0.01)$ and Single Repeat $(\mathrm{t}(11)=2.8$, $P<0.05)$ trials, patients with hippocampal damage were not impaired relative to controls in their performance on Re-paired trials $(\mathrm{t}(11)=1.4, P=0.2)$. In addition, an interaction between subject group and trial type was observed in a $2 \times 2$ repeated measures ANOVA involving only the Single Repeat and Re-paired trials $(\mathrm{F}(1,11)=8.6, P<0.05)$. Whether this finding represents a true sparing of performance on the Re-paired trials or is the result of compression due to a floor effect, the finding on Re-paired trials is inconsistent with a selective impairment in the associative component of declarative memory.

\section{EXPERIMENT 3}

In Experiments 1 and 2, a continuous recognition memory task was used to assess both memory for the individual components of an item and memory for the association between these components. In neither experiment were patients with hippocampal damage differentially impaired for the associative component of recognition memory relative to recognition memory for single items. Reduction in accuracy for the Single Repeat stimuli (in which one of the two components of the item had been previously encountered) was at least as large as the reduction in accuracy for the Re-paired stimuli (in which both components of the items had been encountered previously). 

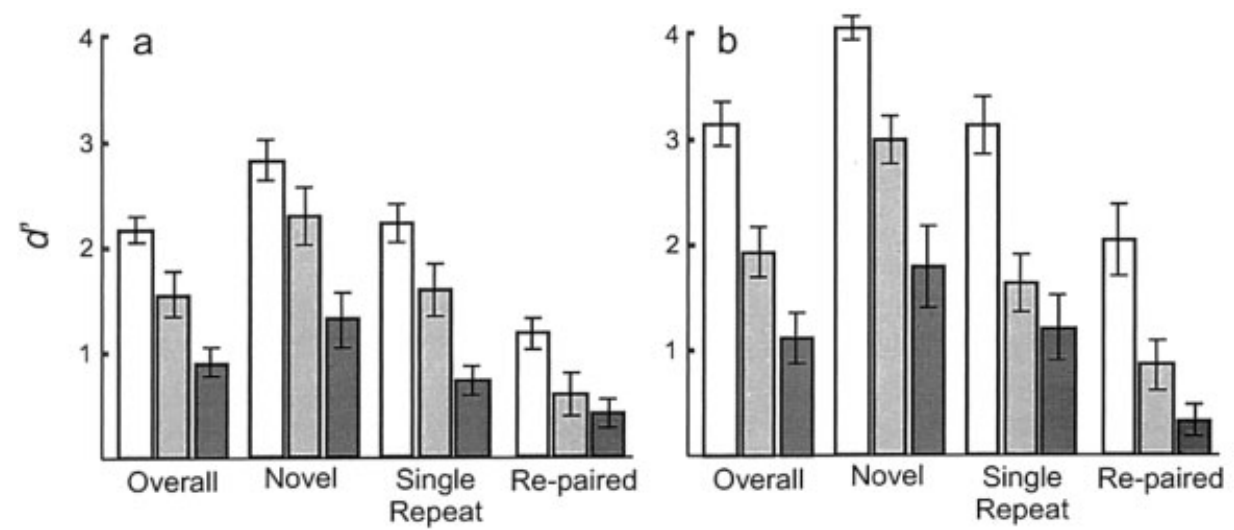
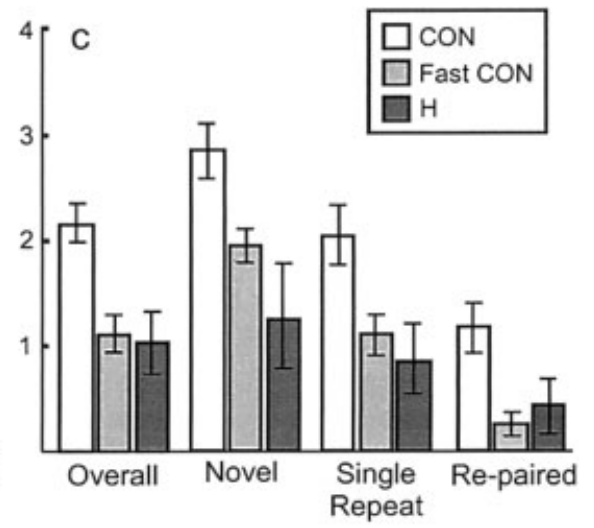

FIGURE 4. Data from Experiments 3 (a), 4 (b), and 5 (c). In each panel, overall discriminability $\left(d^{\prime}\right)$ scores (Overall) were calculated from the hit rate (probability of responding "yes" to true repetitions) and the overall false alarm rate (probability of responding "yes" to all other items) for age-matched controls ( $\mathrm{CON}$; $n=8)$, age-matched controls whose response time was limited to $2 s($ Fast $\mathrm{CON} ; \mathrm{n}=8)$, and patients with damage to the hippocampal region $(H ; n=5)$. In

In both Experiments 1 and 2, a single stimulus was presented. Although the items were always composed of two parts (i.e., two syllables), it remains possible that the items were treated as single entities rather than as an association between two component parts. In Experiments 3-5, we made more direct attempts to encourage participants to view each item as involving an association between two components. In Experiment 3, we presented two monosyllabic words in different locations on the screen, each displayed on a separate object resembling a three-dimensional brick (Fig. 2c).

In addition, to understand how the performance of controls would decline as the task was made more difficult, an additional group of controls was tested in Experiments 3-5. For this group (Fast CON), the test was not self-paced, but a 2-s limit on response time was imposed in an effort to lower performance scores. If the overall performance of controls were similar to that of the patients, it might be easier to observe a differential impairment on Re-paired trials.

\section{Method}

Experiment 3 was identical in structure to Experiments 1 and 2. However, instead of presenting two monosyllabic words as a single pseudo-word (as in Experiment 2) the two monosyllabic words were presented in isolation. Each word was presented in white letters on a red, three-dimensional rectangle that resembled a brick (Fig. 2c). On each trial, participants were asked to decide whether the entire display was one that had been presented previously on the list. Two new groups of controls also participated. One group of eight controls (CON, four males and four females) was given the same test that was administered to the amnesic patients. The other group of controls (Fast CON) was given the same test, but with a limit of $2.0 \mathrm{~s}$ imposed on their response times in an effort to reduce accuracy. Both control groups were matched to the patients with respect to age $(\mathrm{CON}=67.8$, Fast $\mathrm{CON}=65.1)$ and education $(\mathrm{CON}=16$, Fast $\mathrm{CON}=17.1)$. addition, $d^{\prime}$ scores were calculated using the hit rate and each of the three possible false alarm conditions: entirely novel stimuli (Novel), stimuli in which one component had been previously presented (Single Repeat), and novel stimuli in which both components had been previously presented but not together (Re-paired). Impairments were observed in the hippocampal patients across all trial types, not only on the Re-paired trials that assess associative memory.

\section{Results}

Figure 4 a shows the $\mathrm{d}^{\prime}$ scores for the entire test (Overall) and $\mathrm{d}^{\prime}$ scores based on each of the three potential sources of false alarms (false alarm rates for each condition appear in Table 2). Overall, the patients with hippocampal damage were impaired relative to the CON group $(\mathrm{t}(11)=6.0, P<0.001)$. A repeated measures ANOVA comparing performance of the self-paced controls (CON) and the patients with hippocampal damage revealed an interaction between subject group and trial type $(\mathrm{F}(2,22)=7.9$, $P<0.005)$. However, as in the case of Experiment 2, this interaction resulted from a relative sparing of performance on the Repaired trials. Thus, the patients with hippocampal damage were impaired relative to the CON group on all three trial types (all $P$ values $<0.01$ ), but their impairment was less severe for the Repaired trials than for either the Novel trials (interaction of group $X$ trial type, $\mathrm{F}(1,11)=7.0, P<0.05)$ or the Single Repeat trials (interaction of group $\times$ trial type, $\mathrm{F}(1,11)=21.3, P<0.005$ ). Again, whether this finding represents a true sparing of performance on the Re-paired trials or is the result of compression due to a floor effect, the finding for Re-paired trials is inconsistent with a selective impairment in the associative component of declarative memory.

The fast control group (Fast $\mathrm{CON}$ ) was impaired relative to the CON group (overall $\mathrm{d}^{\prime} ;(\mathrm{t}(14)=2.3, P<0.05)$ and did not differ reliably from the patients $(\mathrm{t}(11)=2.0, P>0.05)$. While imposing a limit on response time did reduce the accuracy of the control group and made the scores of this group more similar to those of the patients, the pattern of performance differed between the two groups (interaction of group $\times$ trial type, $(\mathrm{F}(2,22)=8.3, P<$ 0.005). Specifically, the Fast CON group performed better than the patients on the Novel trials $(\mathrm{t}(11)=2.5, P<0.05)$ and on the Single Repeat trials $(\mathrm{t}(11)=2.6, P<0.05)$, but not on the Repaired trials $(\mathrm{t}(11)=0.6, P=0.5)$. Indeed, relative to the Fast $\mathrm{CON}$ group, the patients were less impaired on the Re-paired trials 
than on either the Novel trials $(\mathrm{F}(1,11)=9.1, P<0.05)$ or the Single Repeat trials $(\mathrm{F}(1,11)=26.0, P<0.001)$. From these observations, one might conclude that imposing a time limit on the response times of controls was more effective at impairing performance on the Re-paired trials than was damage to the hippocampus.

\section{EXPERIMENT 4}

In Experiment 3, we encouraged participants to treat the items in a continuous recognition memory test as composed of two distinct components (Fig. 2c). No evidence was found that patients with hippocampal damage were especially impaired in the ability to form associations between the items that were studied. Patients with hippocampal damage exhibited impaired recognition accuracy on Novel trials, Single Repeat trials, as well as on Re-paired trials. Further, in the comparison between Single Repeat trials and Re-paired trials, the impairment was less severe for the Re-paired trials than for the Single Repeat trials. In addition, when the overall performance of controls and patients was made more similar (by imposing a limit on response time for the Fast CON group), no impairment was observed on Re-paired trials, even though performance remained impaired on Novel and Single Repeat trials. Experiments $1-3$ assessed the ability of patients with hippocampal damage to form associations that involved verbal material. To test the generality of our findings, Experiments 4 and 5 involved pictorial material.

\section{Method}

Experiment 4 was identical in structure to the previous experiments. The components of each display were two colored photographs of common objects (Fig. 2d). The same two groups of eight controls from Experiment 3 also participated in Experiment 4.

\section{Results}

Figure $4 \mathrm{~b}$ shows the $\mathrm{d}^{\prime}$ scores for the entire test (Overall) and $\mathrm{d}^{\prime}$ scores based on each of the three potential sources of false alarms (false alarm rates for each condition appear in Table 2). Overall, the patients with hippocampal damage were impaired relative to the CON group $(\mathrm{t}(11)=6.0, P<0.001)$. The Fast $\mathrm{CON}$ group was also impaired relative to the CON group $(\mathrm{t}(14)=3.6, P<$ $0.005)$ and performed marginally better than the patient group $(\mathrm{t}(11)=2.1, P=0.051)$. A repeated measures ANOVA comparing performance of the $\mathrm{CON}$ group to the patient group revealed no interaction between subject group and trial type $(\mathrm{F}(2,22)=$ $1.1, P=0.35)$. Likewise, a repeated measures ANOVA comparing performance of the Fast CON group to the patient group also revealed no significant interaction between subject group and trial type $(\mathrm{F}(2,22)=3.2, P=0.06)$. The source of the trend in these data was that a somewhat larger impairment occurred on the Novel trials. Thus, there was no suggestion in the data that the patients were especially disadvantaged on Re-paired trials.

\section{EXPERIMENT 5}

The results of Experiment 4 were similar to those of Experiments 1-3. Patients with hippocampal damage exhibited an overall impairment on the continuous recognition task, but their impairment was not differentially apparent on the Re-paired trials that were designed to assess the associative component of declarative memory. In Experiment 5, we tried to increase the requirement for associative memory by using items from two distinct categories: faces and houses. Thus, each display in Experiment 5 consisted of a picture of a house and a portrait of a person (Fig. 2e).

\section{Method}

Experiment 5 was identical in structure to the previous experiments. One component of each display was a colored picture of a house, and the other component was a colored portrait of a person (Fig. 2e). The same two groups of eight controls from Experiments 3 and 4 also participated in Experiment 5.

\section{Results}

Figure $4 \mathrm{c}$ shows the $\mathrm{d}^{\prime}$ scores for the entire test (Overall) and $\mathrm{d}^{\prime}$ scores based on each of the three potential sources of false alarms (false alarm rates for each condition appear in Table 2). Overall, the patients with hippocampal damage were impaired relative to the CON group $(\mathrm{t}(11)=3.0, P<0.05)$. A repeated measures ANOVA comparing performance of the CON group and the patients with hippocampal damage revealed an interaction between subject group and trial type $(\mathrm{F}(2,22)=6.1, P<0.01)$. However, as in Experiments 2 and 3, this interaction resulted from a relative sparing of performance on the Re-paired trials. Thus, the patients were impaired relative to the CON group on the Novel trials $(\mathrm{t}(11)=3.0, P<0.05)$ and on the Single Repeat trials $(\mathrm{t}(11)=$ 2.5, $P<0.05)$, and fell just short of significance on Re-paired trials $(\mathrm{t}(11)=2.1, P=0.06)$. Further, their impairment relative to the $\mathrm{CON}$ group was less severe for the Re-paired trials than for the Novel trials (interaction of group $\times$ trial type, $\mathrm{F}(1,11)=8.1, P<$ 0.05 ) or for the Single Repeat trials (interaction of group $\times$ trial type, $\mathrm{F}(1,11)=9.0, P<0.05)$. Again, whether this finding represents a true sparing of performance on the Re-paired trials or is the result of compression due to a floor effect, the finding for Re-paired trials is inconsistent with a selective impairment in the associative component of declarative memory.

The Fast CON group was impaired overall relative to the selfpaced CON group $(\mathrm{t}(14)=3.4, P<0.005)$ and performed at about the same overall level as the patients $(\mathrm{t}(11)=0.2, P=0.8)$. While imposing a limit on response time did match the overall accuracy of the Fast CON group and the patient group, the pattern of performance differed between the two groups (interaction of group $\times$ trial type, $\mathrm{F}(2,22)=4.6, P<0.05)$. The Fast $\mathrm{CON}$ group performed slightly (but not significantly) better than the patients with hippocampal damage on the Novel and Single Repeat trials but slightly worse than the patients on the Re-paired trials. 


\section{DISCUSSION}

Five recognition memory tasks were administered to five amnesic patients with damage to the hippocampal region. Each experiment used the same continuous recognition paradigm employed by Kroll et al. (1996) in which participants were shown stimuli with two components (Fig. 2) and were asked to respond "yes" when a previously encountered stimulus appeared. The two-component stimuli could appear in one of four conditions: novel stimuli that had not appeared earlier in the list (Novel), novel stimuli that shared a single component with a previously encountered stimulus (Single Repeat), novel stimuli in which both components had appeared in different previously encountered stimuli (Repaired), or stimuli that had been previously encountered (True repetition). Experiment 1 used the same disyllabic nouns as Kroll et al. (1996), and Experiments 2-5 attempted to increase progressively the degree to which associative memory would be used to perform the task.

In all five experiments, the patients with damage to the hippocampal region exhibited impaired recognition memory performance. Critically, the impairment was not limited to, or even disproportionately weighted towards the Re-paired trials, but extended relatively evenly across all trial types. Indeed, where differential effects were observed (Experiments 2, 3, and 5), performance was less impaired on the Re-paired trials than on the other trial types. Although this difference in degree of impairment was sometimes statistically reliable, we do not suggest that damage to the hippocampal region selectively spares the associative component of declarative memory. Performance on the Re-paired trials may sometimes (in Experiments 2, 3, and 5; not in Experiments 1 and 4) have been low enough (even for controls) that the $d^{\prime}$ scores were compressed and a larger impairment was thereby somewhat obscured. In any case, it is clear that in this series of experiments, damage to the hippocampal region did not selectively impair the associative or conjunctive component of declarative memory.

Although all five amnesic patients have damage to the hippocampal region (the hippocampus proper, the dentate gyrus, and the subiculum) two of the patients (H.C., and P.H.) have damage that extends beyond the hippocampal region into the parahippocampal gyrus. Therefore, it is possible that a selective deficit in the Repaired condition was present in the three patients whose damage is limited to the hippocampal region. Figure 5 shows the combined results across all five experiments for the healthy volunteers $(\mathrm{CON})$, the three patients with damage limited to the hippocampal region $(\mathrm{H})$, and the two patients with damage to both the hippocampal region and the parahippocampal gyrus $(\mathrm{H}+)$. A repeated-measures ANOVA was used to examine the effects of stimulus condition (Novel, Single-Repeat, or Re-paired), experimental paradigm (Experiments 1-5), and patient group ( $\mathrm{H}$ or $\mathrm{H}+$ ) on $\mathrm{d}^{\prime}$ scores. No reliable main effect of patient group or interactions with the patient group were observed (all $\mathrm{F}$ values $<0.9$ ). A separate ANOVA that assessed the effects of experimental paradigm (Experiments 1-5) and patient group on the $\mathrm{d}^{\prime}$ score for just the Re-paired condition also revealed no effects of patient

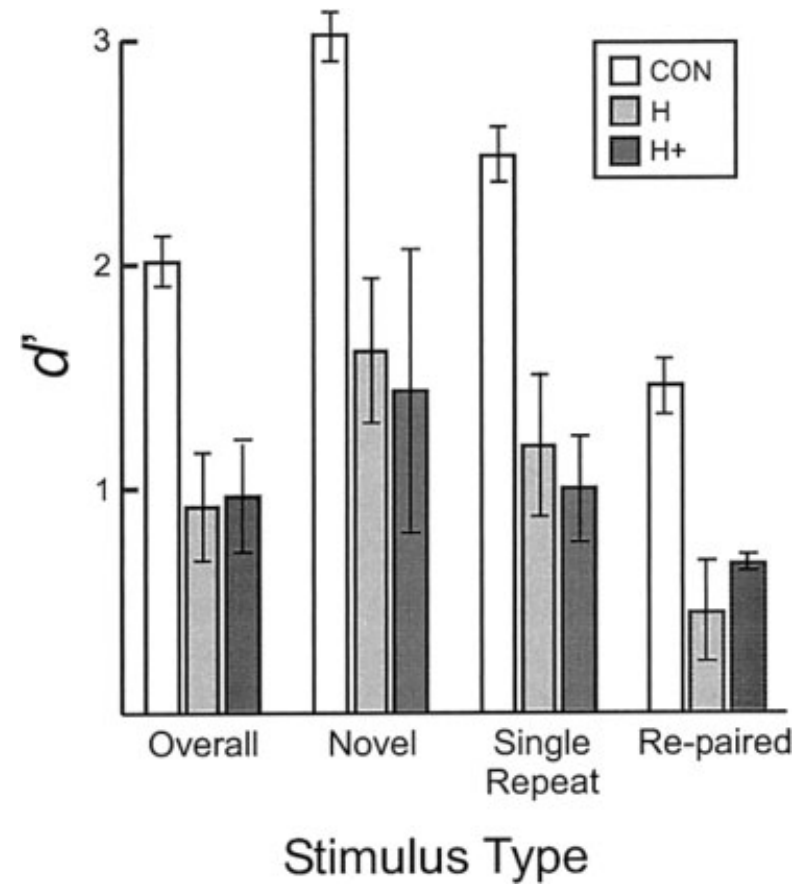

FIGURE 5. Data combined across all five experiments for the age-matched controls $(\mathrm{CON} ; \mathrm{n}=8)$, the three patients with damage limited to the hippocampal region $(\mathrm{H})$, and the two patients with damage to both the hippocampal region and the parahippocampal gyrus $(\mathbf{H}+)$.

group or interactions involving the patient group (all $\mathrm{F}$ values $<0.6)$.

These results are inconsistent with reports that have proposed a specific role for the human hippocampus in associative memory. First, the results differ from those obtained by Kroll et al. (1996). While Kroll et al. (1996) observed an impairment only on the Re-paired (associative) trials, we observed impairments across all trial types that were not disproportionately weighted towards the Re-paired trials. This was true both in an experiment designed to replicate the study by Kroll et al. (1996) (Experiment 1) and in additional experiments designed to emphasize more strongly the associative component of the task (Experiments 2-5). Comparisons of our results from Experiment 1 with those of Kroll et al. (1996) (Fig. 1 vs Fig. 3a) indicate that the controls in our study scored higher overall than those tested by Kroll et al. (1996) and that our amnesic patients scored lower overall than the patients tested by Kroll et al. (1996). Further, in our study, the patients were impaired on those trials that would constitute a traditional recognition memory test (Novel trials), whereas on the same trials the patients tested by Kroll et al. (1996) performed numerically better than controls. If the performance of the controls were elevated (or the performance of the patients were reduced), such that the patients scored at least as poorly as the controls on the Novel items, then a reliable impairment on the Single Repeat trials might have been observed in the earlier study and evidence for a selective impairment on Re-paired (associative) trials would have been weakened. Although we cannot be sure why our results differed from those obtained by Kroll et al. (1996), any inadvertent mis- 
match of the controls to the patients in the Kroll et al. (1996) study might explain the discrepancy in the results. In addition, we should note again that the patients in the two studies have different lesions. This, too, is a potential explanation for the discrepancy in the results.

Findings from functional neuroimaging have also been taken to support the view that the hippocampus is specifically involved in associative memory. In a study using position emission tomography (PET), Henke et al. (1997) observed greater hippocampal activity during an associative learning task (memorizing facehouse pairs) than during the learning of single items (faces or houses) or during retrieval. However, the same result was obtained in the parahippocampal gyrus. Similarly, in a subsequent study, Henke et al. (1999) again reported greater activity during associative learning than during nonassociative learning in both the hippocampal region and in the parahippocampal gyrus. Further, in an fMRI study of recognition memory, Yonelinas et al. (2001) reported greater activity during associative than nonassociative recognition in both the hippocampus and the parahippocampal gyrus. Accordingly, although these data support a role for the medial temporal lobe in associative memory, they do not differentiate between the hippocampus and parahippocampal gyrus in this regard.

Studies involving the Remember/Know paradigm (Tulving, 1985) are also pertinent to this discussion, if one assumes that "Remember" responses (when participants report that they actually remember the study episode) are more associative in nature than "Know" responses (when participants report that they know only that the item was recently encountered, and cannot recollect the study episode itself). We should note that one difficulty with this assumption is that Remember responses might differ from Know responses, not because Remember responses are more associative, but because Remember responses reflect more memory content than Know responses.

In any case, in one recent study (Eldridge et al., 2000), greater activity was observed for Remember responses than for Know responses in both the hippocampal region and the parahippocampal gyrus (Fig. 1, Table 1, Eldridge et al, 2000). Thus, these findings also do not support a distinction between the hippocampal region and the parahippocampal gyrus with respect to associative memory. The observation in that study that Know responses yielded no greater activation in the hippocampal region than correct rejections of the unfamiliar foil items likely resulted from elevated activity associated with encoding the unfamiliar stimuli (for evidence that activation associated with encoding unfamiliar stimuli can work in opposition to finding activation associated with hits minus correct rejections, see Stark and Squire, 2001a). Finally, the observation by Eldridge et al. (2000) that neither Know responses nor correct rejections were associated with activity above the baseline condition likely was due to the baseline condition they opted to use, namely a 20 -s-long period of fixation between trials. In actuality, fixation and rest conditions are relatively active conditions and are associated with significantly more medial temporal lobe activity than a number of more "mindless" conditions that involve, for example, repetitive performance of simple tasks (Stark and Squire, 2001b). Therefore, when a task activates the medial tem- poral lobe as much as rest or fixation, one cannot conclude that the task does not activate the medial temporal lobe to a meaningful extent.

Electrophysiological data have also been taken in support of the hypothesis that the hippocampus is particularly involved in associative forms of memory. That the activity of hippocampal neurons code for conjunctions or associations of features has been well established (for review, see Eichenbaum, 2000; Suzuki and Eichenbaum, 2000; Brown and Aggleton, 2001). However, it is also the case that the activity of neurons in the parahippocampal gyrus can also code for conjunctions or associations of features. For example, Fried et al. (1997) recorded from human hippocampal and entorhinal neurons while patients were given encoding tasks and recognition memory tasks with faces that varied in facial expression and gender. Fried et al. (1997) reported conjunctive responses (e.g., neurons responsive to specific combinations of expression and gender) not only in the hippocampus, but in the entorhinal cortex as well. Conversely, while many hippocampal neurons code for conjunctions of stimuli, conjunctive coding is not universally observed. For example, Wood et al. (1999) recorded activity from rat hippocampus (CA1 and CA3) as animals performed a continuous, odor-guided, non-matching-to-sample recognition memory task. The activity of cells correlated with the position of the sample (place cells), the odor presented, whether the trial was a match or nonmatch, whether the rat was approaching the sample, and four different conjunctions of these factors. Importantly, within the $56 \%$ of task-relevant cells that had only nonspatial correlates (one could argue that spatial factors are inherently associative), an overwhelming 96\% responded only to single task features. Although such data cannot conclusively demonstrate nonassociative codes in the hippocampus (a cell might have responded to conjunctions between the tested feature and some feature not explicitly tested), the data are nevertheless consistent with the view that hippocampal neurons respond to single stimulus features. In addition, in the study by Wood et al. (1999), a number of hippocampal neurons did exhibit the stimulus-selective behavior observed in perirhinal cortical neurons that has been attributed to nonassociative memory, e.g., detection of familiarity (Brown and Aggleton, 2001).

Studies of transitive inference and transverse patterning problems have sometimes been taken in support of a restricted version of the associative hypothesis - that the hippocampus plays a specific role in memory for complex relationships (but not in memory for simple associations). In the transitive inference problem, a set of pair-wise discriminations are taught (e.g., $\mathrm{A}>\mathrm{B}, \mathrm{B}>\mathrm{C}, \mathrm{C}>\mathrm{D}$, $\mathrm{E}>\mathrm{F}$ ), and testing then assesses whether the hierarchical structure of the stimuli (knowledge that $\mathrm{B}>\mathrm{D}$ ) has been learned, or whether simply a series of pair-wise discriminations has been learned. Dusek and Eichenbaum (1997) reported that rats with either fornix transection or conjoint perirhinal and entorhinal lesions were able to learn the pair-wise discriminations normally, but failed to make the transitive inference that $\mathrm{B}>\mathrm{D}$. In a separate experiment with the transverse patterning problem $(A>B, B>C, C>A)$, Dusek and Eichenbaum (1998) reported that rats with fornix or perirhinal/entorhinal lesions could learn the pair-wise discriminations when they were presented in sequential blocks, but were 
impaired when testing continued with randomly presented pairs (for similar results, see Alvarado and Rudy, 1995a,b).

In a recent PET study of the transitive inference problem (Nagode and Pardo, 2002), less activity was observed in the region of the hippocampus during training on blocks of independent pairwise discriminations (e.g., $\mathrm{A}>\mathrm{B}, \mathrm{C}>\mathrm{D}, \mathrm{E}>\mathrm{F}$ ) than during subsequent training on blocks of the "bridging" pair-wise discriminations that could then support transitive inference (e.g., $\mathrm{B}>\mathrm{C}$, $\mathrm{D}>\mathrm{E}$ ). It should be noted that the ability to localize activity within the hippocampus is limited given the resolution of PET and the lack of structural MRI scans from the participants.

These data do not provide unambiguous support for a specific role for the hippocampus in learning complex relationships. First, although rats with fornix transection, hippocampal lesions, or perirhinal/entorhinal lesions can learn individual pair-wise discriminations at normal rates (Bunsey and Eichenbaum, 1996; Dusek and Eichenbaum, 1997, 1998), amnesic patients with hippocampal lesions are unequivocally impaired at learning unrelated stimulus pairs (Squire and Shimamura, 1986). Indeed, paired-associate learning tasks are considered to be among the most sensitive measures of impaired memory (Erickson and Scott, 1977). Rats with lesions of the hippocampus or related structures may be able to learn discriminations as tasks of habit learning that depend on the integrity of the caudate nucleus (for discussion, see Teng et al., 2000). Second, Reed and Squire (1999) gave the transverse patterning problem to amnesic patients, including three patients who participated in the present study (A.B., L.J., and P.H.). The patients were impaired at acquiring the transverse patterning problem $(A>B$, $\mathrm{B}>\mathrm{C}, \mathrm{C}>\mathrm{A}$ ), but were also impaired at acquiring three independent pair-wise discriminations $(\mathrm{D}>\mathrm{E}, \mathrm{F}>\mathrm{G}, \mathrm{H}>\mathrm{I})$. Further, for both amnesic patients and controls, learning the transverse patterning problem appeared to be about as difficult as learning six independent pair-wise discriminations. Thus, for humans, the transverse pattering problem is simply a set of three discrimination problems that are difficult to memorize because they contain overlapping elements that cause interference. Thus, rats and humans approach the discrimination problems differently, and it is therefore difficult to generalize from findings of discrimination performance in rats. Finally, given that discrimination learning in humans is hippocampus-dependent, it is unclear why Nagode and Pardo (2002) did not observe any activity in any medial temporal lobe structure, in any of their contrasts, during components of their tasks that involved simple discrimination learning.

From our results and the results just reviewed, we conclude that (1) the capacity for acquiring associative components of memory is not the special province of the hippocampal region but is the province of the parahippocampal gyrus as well; and (2) although associative remembering might sometimes activate medial temporal lobe structures more than single-item remembering (e.g., because more is being recalled), the hippocampal region is essential for both kinds of memory. Although functional specialization likely exists within the medial temporal lobe memory system, the present data suggest that any division of labor involving associative or conjunctive memory cannot be absolute (for a similar view that draws on electrophysiological data, see Suzuki and Eichenbaum, 2000). Indeed, it has been emphasized previously that all traditional single-item recognition tasks are associative in the sense that study items must be associated with the learning context (Stark and Squire, 2000b; Zola and Squire, 2000; Stark and Squire, 2001a). Thus, we suggest that recent proposals that the hippocampal region is important for relational memory, declarative memory, or conjunctive memory are all on target, but that incorrect predictions have sometimes been made about how selective lesions of the hippocampal region should affect memory, e.g., that hippocampal lesions should affect only the formation of explicit associations, or only recollection (not familiarity), or only episodic memory (not semantic memory).

Recently, O'Reilly and colleagues (O'Reilly and Rudy, 2000; O'Reilly and Rudy, 2001; Norman and O'Reilly, in press) have reconsidered the configural association theory of hippocampal function (Sutherland and Rudy, 1989) in light of the computational principles described by McClelland et al. (1995). O'Reilly and colleagues suggested that the interaction between the hippocampal region and the parahippocampal gyrus is best understood by adopting a computationally explicit and biologically motivated approach. They also argue against an absolute division of labor between the hippocampal region and the parahippocampal gyrus with respect to associative or conjunctive memory. Instead, they propose that moderate differences in the learning rate (amount of change in connection strengths with each learning episode) and in the sparseness of internal representations (proportion of the units that are "on" at any one time, and therefore the degree to which different patterns of activity overlap) between the hippocampal region and parahippocampal gyrus will result in different, but not entirely distinct, functions. These differences make the hippocampal region better suited to support some tasks of declarative memory than the parahippocampal gyrus (e.g., tasks that require rapid, incidental conjunctive learning). Thus, the proposed model is a graded one in which both the hippocampal region and the parahippocampal gyrus are capable of and important for complex associative learning. In this model, whether a lesion of the hippocampus would result in the pattern of data observed in our study is not entirely clear. Nevertheless, by virtue of being both computationally explicit and biologically motivated, the exploration of such models would seem to provide a more promising approach to furthering our understanding of hippocampal and parahippocampal function than simple dichotomies such as associative versus nonassociative memory, episodic versus semantic memory, or recollection versus familiarity.

\section{Acknowledgments}

The authors thank Shauna Stark for her assistance with programming, data collection, and data analysis. In addition we thank Jennifer Frascino and Joyce Zouzounis for their assistance in data collection. This research was supported by the Medical Research Service of the Department of Veterans Affairs, National Institute of Mental Health Grants MH24600 and MH12278, the National Alliance for Research on Schizophrenia and Depression (NARSAD), and the Metropolitan Life Foundation. 


\section{REFERENCES}

Aggleton JP, Brown MW. 1999. Episodic memory, amnesia, and the hippocampal-thalamic axis. Behav Brain Sci 22:425-444.

Alvarado M, Rudy JW. 1995a. A comparison of kainic acid plus colchicine and ibotenic acid-induced hippocampal formation damage on four configural tasks in rats. Behav Neurosci 109:1052-1062.

Alvarado M, Rudy JW. 1995b. Rats with damage to the hippocampalformation are impaired on the transverse-pattering problem but not on elemental discriminations. Behav Neurosci 109:204-211.

Baddeley A, Vargha-Khadem F, Mishkin M. 2001. Preserved recognition in a case of developmental amnesia: implications for the acquisition of semantic memory? J Cogn Neurosci 13:357-369.

Broadbent NJ, Clark RE, Zola SM, Squire LR. 2002. The medial temporal lobe and memory. In: Squire LR, Schacter DL, editors. The neuropsychology of memory. 3rd Ed. New York: Guilford. p 3-23.

Brown MW, Aggleton JP. 2001. Recognition memory: what are the roles of the perirhinal cortex and hippocampus? Nat Rev Neurosci 2:51-61.

Bunsey M, Eichenbaum H. 1996. Conservation of hippocampal memory function in rats and humans. Nature 379:255-257.

Dusek J, Eichenbaum H. 1997. The hippocampus and memory for orderly stimulus relations. Proc Natl Acad Sci U S A 94:7109-7114.

Dusek J, Eichenbaum H. 1998. The hippocampus and transverse patterning guided by olfactory cues. Behav Neurosci 112:762-771.

Eichenbaum H. 2000. A cortical-hippocampal system for declarative memory. Nat Rev Neurosci 1:41-50.

Eichenbaum H, Otto T, Cohen NJ. 1994. Two component functions of the hippocampal memory system. Behav Brain Sci 17:449-517.

Eldridge LL, Knowlton BJ, Furmanski CS, Bookheimer SY, Engel SA. 2000. Remembering episodes: a selective role for the hippocampus during retrieval. Nat Neurosci 3:1149-1152.

Erickson R, Scott M. 1977. Clinical memory testing: a review. Psychol Bull 84:1130-1149.

Fried I, MacDonald K, Wilson C. 1997. Single neuron activity in human hippocampus and amygdala during recognition of faces and objects. Neuron 18:753-765.

Henke K, Buck A, Weber B, Wieser HG. 1997. Human hippocampus establishes associations in memory. Hippocampus 7:249-256.

Henke K, Weber B, Kneifel S, Wieser HG, Buck A. 1999. Human hippocampus associates information in memory. Proc Natl Acad Sci U S A 96:5884-5889.

Holdstock JS, Mayes AR, Roberts N, Cezayirli E, Isaac SL, O’Reilly RC, Norman KA. 2002. Under what conditions is recognition spared relative to recall after selective hippocampal damage in humans? Hippocampus 12:341-351.

Kroll NEA, Knight RT, Metcalfe J, Wolf ES, Tulving E. 1996. Cohesion failure as a source of memory illusions. J Mem Lang 35:176-196.

Manns JR, Squire LR. 1999. Impaired recognition memory on the Doors and People Test after damage limited to the hippocampal region. Hippocampus 9:495-499.

Manns JR, Squire LR. 2001. Perceptual learning, awareness, and the hippocampus. Hippocampus 11:776-782.

McClelland JL, McNaughton BL, O'Reilly RC. 1995. Why there are complementary learning systems in the hippocampus and neocortex: insights from the successes and failures of connectionist models of learning and memory. Psychol Rev 102:419-457.

Nagode J, Pardo J. 2002. Human hippocampal activation during transitive inference. NeuroReport 13:939-944.

Norman KA, O'Reilly RC. in press. Modeling hippocampal and neocortical contributions to recognition memory: a complementary learning systems approach. Psych Rev.

O'Reilly RC, Rudy JW. 2000. Computational principles of learning in the neocortex and hippocampus. Hippocampus 10:389-397.

O'Reilly RC, Rudy JW. 2001. Conjunctive representations in learning and memory: principles of cortical and hippocampal function. Psychol Rev 108:311-345.

Reed JM, Squire LR. 1997. Impaired recognition memory in patients with lesions limited to the hippocampal formation. Behav Neurosci 111: 667-675.

Reed JM, Squire LR. 1999. Impaired transverse patterning in human amnesia is a special case of impaired memory for two-choice discrimination tasks. Behav Neurosci 113:3-9.

Rempel-Clower NL, Zola SM, Squire LR, Amaral DG. 1996. Three cases of enduring memory impairment after bilateral damage limited to the hippocampal formation. J Neurosci 16:5233-5255.

Squire LR, Shimamura AP. 1986. Characterizing amnesic patients for neurobehavioral study. Behav Neurosci 100:866-877.

Stark CEL, Squire LR. 2000a. Recognition memory and familiarity judgments in severe amnesia: no evidence for a contribution of repetition priming. Behav Neurosci 114:459-467.

Stark CEL, Squire LR. 2000b. Functional magnetic resonance imaging (fMRI) activity in the hippocampal region during recognition memory. J Neurosci 20:7776-7781.

Stark CEL, Squire LR. 2001a. Simple and associative recognition memory in the hippocampal region. Learn Mem 8:190-197.

Stark CEL, Squire LR. 2001b. When zero is not zero: the problem of ambiguous baseline conditions in fMRI. Proc Natl Acad Sci U S A 98:12760-12766.

Sutherland RJ, Rudy JW. 1989. Configural association theory: the role of the hippocampal formation in learning, memory, and amnesia. Psychobiology 17:129-144.

Suzuki WA, Eichenbaum H. 2000. The neurophysiology of memory. Ann N Y Acad Sci 911:175-191.

Teng E, Stefanacci L, Squire LR, Zola SM. 2000. Contrasting effects on discrimination learning after hippocampal lesions and conjoint hippocampal-caudate lesions in monkeys. J Neurosci 20:3853-3863.

Tulving E. 1985. Memory and consciousness. Can J Psychol 26:1-12.

Vargha-Khadem F, Gadian DG, Watkins KE, Connelly A, Van Praesschen W, Mishkin M. 1997. Differential effects of early hippocampal pathology on episodic and semantic memory. Science 277:376-380.

Wood E, Dudchenko P, Eichenbaum H. 1999. The global record of memory in hippocampal neural activity. Nature 397:613-616.

Yonelinas A, Hopfinger J, Buonocore M, Kroll N, Baynes K. 2001. Hippocampal, parahippocampal and occipital-temporal contributions to associative and item recognition memory: an fMRI study. NeuroReport 12:359-363.

Zola SM, Squire LR. 2000. The medial temporal lobe and the hippocampus. In: Tulving E, Craik FIM, editors. The Oxford handbook of memory. New York: Oxford University Press. p 501-520. 